\title{
Transcutaneous Bilirubinometry: A Noninvasive Tool for Studying Newborn Jaundiced Rats Before and After Exposure to Light
}

\author{
HENDRIK J. VREMAN, RONALD J. WONG, MIU L. CHAN, BETTY W.Y. YOUNG, AND DAVID K. STEVENSON
}

Department of Pediatrics [H.J.V., R.J.W., M.L.C., D.K.S.], Stanford University School of Medicine, Stanford, California 94305; Department of Pediatrics [M.L.C., B.W.Y.Y.], Pamela Youde Nethersole Eastern Hospital Hong Kong, China

\begin{abstract}
The homozygous Gunn rat is the most frequently used animal model for the study of neonatal jaundice. We evaluated the applicability of noninvasive transcutaneous bilirubin $(\mathrm{TcB})$ measurements as an index of serum total bilirubin (STB) levels in neonatal rats by comparison to invasive STB measurements. TcB measurements were made during the first $96 \mathrm{~h}$ of life with the Model 101 Minolta/Air-Shields Jaundice Meter (JM) and SpectRx BiliCheck System (BC). Measurements with both devices displayed parallel TcB profiles, rapidly rising within $24 \mathrm{~h}$, increasing during the next $6 \mathrm{~h}$, then leveling off after $30 \mathrm{~h}$. Linear regressions for the JM $(n=$ $60)$ were as follows: STB $(\mathrm{mg} / \mathrm{dL})=0.79(\mathrm{JM})-0.01$ (units, $r=$ 0.95 , head); STB $(\mathrm{mg} / \mathrm{dL})=0.82(\mathrm{JM})+1.51$ (units, $r=0.95$, upper back); and STB $(\mathrm{mg} / \mathrm{dL})=0.74(\mathrm{JM})+1.60$ (units, $r=0.91$, lower back). Mean bias \pm imprecision were as follows: $-0.02 \pm 3.99$ $\mathrm{mg} / \mathrm{dL},-0.01 \pm 3.90$, and $0.01 \pm 4.28$ at the head, upper back, and lower back, respectively. For the BC, only lower back measurements were taken, and the regression was as follows: STB $(\mathrm{mg} / \mathrm{dL})$ $=0.77(\mathrm{BC})+1.65 \mathrm{mg} / \mathrm{dL},(r=0.93, n=29)$ with a mean bias \pm imprecision of $-1.08 \pm 3.08 \mathrm{mg} / \mathrm{dL}$. When pups were exposed to light, correlations remained strong but intercepts increased. These results demonstrate that noninvasive $\mathrm{TcB}$ measurements correlate highly with STB in the Gunn rat during the first $96 \mathrm{~h}$ of life and after exposure to light. We conclude that JM measurements at the head and BC at the lower back reflect STB most reliably and consistently. Thus, in addition to being a useful tool for evaluating jaundice in human neonates, TcB methodology can be used successfully for the noninvasive monitoring of jaundice in neonatal Gunn rats pre- and postlight exposure. (Pediatr Res 59: 203-209, 2006)
\end{abstract}

$\mathbf{J}^{\mathrm{a}}$ aundice is a condition characterized by yellowness of the skin and sclerae. It is a consequence of an imbalance between the production and excretion of bilirubin, which ultimately results in the accumulation of excessive bilirubin in circulation (hyperbilirubinemia) and subsequently in skin (jaundice). This syndrome is common during the postnatal period when the human newborn, which has an immature

Received June 17, 2005; accepted September 7, 2005.

Correspondence: Hendrik J. Vreman, Ph.D., Department of Pediatrics, Room S214, Stanford University School of Medicine, Stanford, CA 94305-5208; e-mail: henk. vreman@stanford.edu

Preliminary reports of this study have been presented at the 1999 Society for Pediatric Research in San Francisco, CA, in abstract form and as posters

This work was supported by the National Institutes of Health grants HL68703 and HL58013, the Hess Research Fund, the Mary L. Johnson Research Fund, and the H.M. Lui Research Fund.

DOI: 10.1203/01.pdr.0000196737.73851.8a hepatic bilirubin conjugation and elimination system, is separated from the mother's bilirubin excretion system (1). The condition resolves usually within $10 \mathrm{~d}$ as the liver matures, but persists for newborns with Crigler-Najjar syndrome, who lack the lifelong capability to produce sufficient levels of UGT (2). Although a mild degree of hyperbilirubinemia may be protective for the newborn (3), excessive jaundice, often associated with hemolytic conditions, prematurity (4), and infants of diabetic mothers (5), may lead to life-threatening kernicterus(6).

Developing hyperbilirubinemia is usually diagnosed and monitored through successive, invasive measurements of STB or plasma total bilirubin. (The term STB is frequently used to describe measurements of bilirubin in either serum or plasma. Even though bilirubin measurements in serum and plasma appear to be equivalent, the authors believe that, for the sake of accuracy, the appropriate bilirubin matrix needs to be identified.) Because the removal of blood from newborns is associated with pain, health risks, and financial costs, techniques such as spectrophotometric $\mathrm{TcB}$ measurements (bilirubinometry) have been developed to estimate STB levels noninvasively (7). This methodology has been shown to correlate reasonably well with STB and thus is being used for monitoring jaundice in human newborns $(8,9)$. In nonhuman animals, only cats and dogs have been studied with this technology (10).

The Gunn rat (GR), a mutant Wistar strain first described by Gunn in 1938, is the most frequently used animal model for the study of neonatal jaundice, Crigler-Najjar syndrome, and kernicterus $(11,12)$. Its characteristic nonhemolytic jaundice, an autosomal recessive trait, is the result of a deficiency in hepatic, renal, and gastrointestinal UGT, the enzyme that catalyzes the conjugation of bilirubin with glucuronic acid to form excretable mono- and diconjugated glucuronides (13). Because GR cannot conjugate nor excrete unconjugated bilirubin, they become hyperbilirubinemic. Homozygous (j/j) GR appear normal at birth, but become visibly jaundiced within $16-48 \mathrm{~h}(14,15)$. Heterozygous rats $(\mathrm{J} / \mathrm{j})$ also have reduced UGT levels, but not to the extent that bilirubin excretion is impaired. In GR, STB levels reach a plateau of $<10 \mathrm{mg} / \mathrm{dL}$ when unconjugated bilirubin movement into the skin and

Abbreviations: STB, serum total bilirubin; TcB, transcutaneous bilirubin; UGT, uridine diphosphoglucuronate glucuronosyltransferase 
excretion via the intestine equilibrate with bilirubin production (14).

The UGT deficiency and progressive jaundice found in the homozygous newborn GR mimic the transient deficiency in the bilirubin conjugating ability observed in human newborn infants during the transitional period (14). To fully study the development of jaundice and the factors that affect this process, frequent monitoring of STB concentrations is required. However, this is not feasible in these small animals, which weigh approximately $7 \mathrm{~g}$. It has been shown that the concentration of bilirubin in adult GR skin extracts positively correlates with the STB level in these animals $(r=0.77)(16)$. Thus, the application of a noninvasive means to estimate STB levels would be a useful research tool, for instance, for the study of the in vivo efficacy of metalloporphyrin inhibitors of bilirubin production $(17,18)$ and possibly the efficacy of phototherapy procedures or the in vivo evaluation of phototherapy light sources (unpublished observation).

The objective of this study was to determine whether TcB measurements can be used as an index of STB levels in the neonatal GR and determine the effect of light exposure on these measurements $(19,20)$.

\section{MATERIALS AND METHODS}

Animals. Approval was obtained from the Institutional Animal Care and Use Committee at Stanford University. Newborn homozygous $(\mathrm{j} / \mathrm{j})$ and heterozygous (J/j) GR pups (Harlan Laboratories, Indianapolis, IN), 1-7-d-old were used. These pups were born to female $(J / j)$ GR bred with a $(j / j)$ male. Also, newborn Wistar rats (J/J) (Simonsen Laboratories, Gilroy, CA) were used for reference levels of the postnatal course of STB levels. Pups were housed with their mothers at $23^{\circ} \mathrm{C}$ and kept from direct light since birth. The mothers received food and water ad libitum.

$\boldsymbol{T} \boldsymbol{c B}$ measurements. Two hand-held transcutaneous bilirubinometers, the Minolta/Air-Shields Jaundice Meter (JM, model 101, Minolta Camera Co., Osaka, Japan, Fig. 1A) and the BiliCheck System (BC, SpectRx Inc., Norcross, GA, Fig. $1 B$ ), were used. The latter instrument became available to us after the study had started, thus fewer $(n=29)(\mathrm{j} / \mathrm{j})$ GR pups were studied with this device.

The JM was the first commercially available TcB meter (21). Its accuracy and precision have been well described (22) and it has been used in many clinical studies $(7,8,17)$. The instrument works as follows: when the fiberoptic probe is pressed against the skin, a battery-powered xenon lamp is activated directing a flash of white light at the blanched skin. Reflected light, returned to the unit via a second fiberoptic bundle, is divided by a dichroic filter into

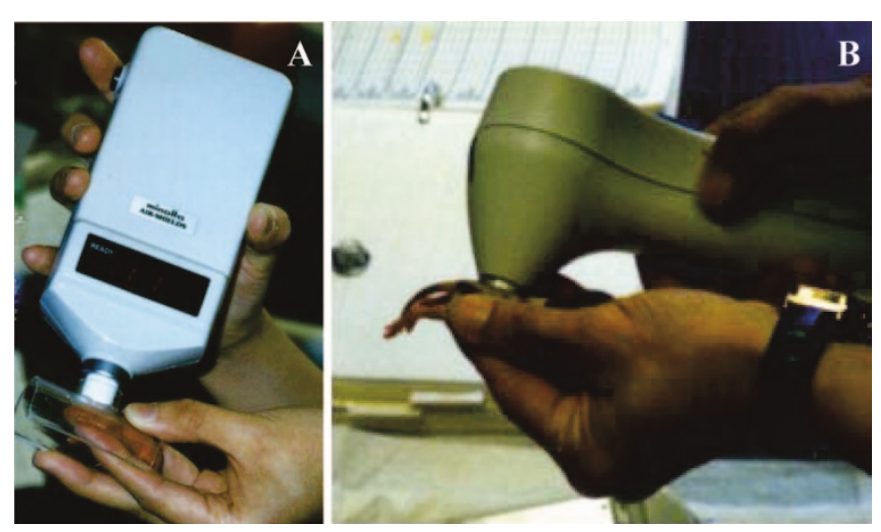

Figure 1. TcB devices used. (A) Minolta/Air-Shields Jaundice Meter (JM), model 101. Readings are taken at the head of a newborn GR pup using a custom-made acrylic jig. Additional measurements were made at the upper and lower back. (B) SpectRx BiliCheck System (BC). Readings could only be taken at the lower back with this device. two component spectra, one of which passes through a blue light filter (maximum absorption at $460 \mathrm{~nm}$ ) and the other through a green filter (maximum absorption at $550 \mathrm{~nm}$ ). Photocells then convert the filtered, reflected light into electronic signals. The intensity of the absorbed $\mathrm{Hb}$ corrected yellow light is obtained as the difference between the absorbance of blue and green light, and is displayed as an arbitrary unit, which has been found to correlate linearly with STB concentrations in humans. A custommade Plexiglas jig was used to protect the pups from excessive pressure $\left(>150 \mathrm{~g} / \mathrm{cm}^{2}\right)$ being exerted during the application of the spring-loaded probe (Fig. 1A). TcB measurements using the JM were made at the back of the head (occiput), upper back in the interscapular region, and lower back over the lumbosacral region. Three consecutive readings were taken at each site and the mean was used.

The BC (Fig. 1B) is a more recently developed device, which operates on similar principles as the JM, but contains more sophisticated optics and electronics for reflectance signal processing. Its design allows for algorithmic corrections for skin components that impact on the bilirubin-related spectral reflectance in newborns, such as dermal type and maturity $(7,8,17)$ and concentrations of melanin and $\mathrm{Hb}(6)$. This instrument is calibrated before each measurement with a probe tip (BiliCal, SpectRx Inc.) that contains standard reference material. This device was designed for use in mixed ethnic populations and with premature infants. Because of the relatively large diameter of the measuring probe $(22 \mathrm{~mm})$, the use of the Plexiglas jig was not necessary, because, while holding the pup, the investigator's fingers absorbed most of the probe's pressure. However, the probe also prevented its reliable application to the head and upper back. Therefore, measurements with the BC were only taken at the lumbosacral (back) region. The BC's algorithm requires that five consecutive measurements be made and the mean value is then displayed as milligrams per deciliter.

Both JM and BC measurements were performed at regular intervals every 2-12 h during the first $96 \mathrm{~h}$ and daily until $168 \mathrm{~h}$ of life or until the animals were killed for STB measurements.

STB measurements. For STB measurements, at least three pups of each genotype and controls were killed by decapitation at the indicated time points after the last TcB measurement. Blood was collected in Microtainer Brand Serum Separator tubes (BD Biosciences, Franklin Lakes, NJ), centrifuged for $1 \mathrm{~min}$ at $13,000 \times g$, and the serum was analyzed for STB with a UB Analyzer (Model UA-1, Arrows Co. Ltd., Osaka, Japan) (24).

Light exposure studies. Four to five randomly selected pups per light source had their TcB measured and were then placed into $17-\mathrm{mL}(55 \times 20 \mathrm{~mm}$ inner diameter) acrylic chambers, which were supplied with air at $20 \mathrm{~mL} / \mathrm{min}$. Chambers with experimental animals were then exposed for $4 \mathrm{~h}$ to $360^{\circ}$ illumination by blue (irradiance $=59 \mu \mathrm{W} / \mathrm{cm}^{2} / \mathrm{nm}$ ), blue-green (irradiance = $30 \mu \mathrm{W} / \mathrm{cm}^{2} / \mathrm{nm}$ ), green (irradiance $=6 \mu \mathrm{W} / \mathrm{cm}^{2} / \mathrm{nm}$ ), and white (irradiance $=$ $11 \mu \mathrm{W} / \mathrm{cm}^{2} / \mathrm{nm}$ ) light-emitting diodes (LED, $n=40$, Nichia Chemical Corp., Anau, Japan), evenly arranged in eight strips of five LED around the circumference of the chamber and operated at 24 VDC and 0.35-0.48 A (Fig. 2A) (25). Additional experimental animals were exposed to $360^{\circ}$ of light produced by one special blue fluorescent (Philips F20T12/BB, irradiance $=55 \mu \mathrm{W} / \mathrm{cm}^{2} / \mathrm{nm}$ ) plus two cool white fluorescent tubes (Sylvania Cool White F20T12/CW, irradiance = $12 \mu \mathrm{W} / \mathrm{cm}^{2} / \mathrm{nm}$ ) or were wrapped in a BiliBlanket Plus (Ohmeda Medical, Columbia, MD) operated at maximum intensity $\left(35 \mu \mathrm{W} / \mathrm{cm}^{2} / \mathrm{nm}\right)($ Fig. $2 A$, far right). Light intensity measurements were made with a BiliBlanket Meter I (Ohmeda Medical), which measures spectral irradiance over a range of 400-520 $\mathrm{nm}$ with peak sensitivity at $470 \mathrm{~nm}$. Control animals were kept in light-shielded chambers. All chambers were air cooled and operated at $23 \pm 2{ }^{\circ} \mathrm{C}$.

Data analysis. TcB readings are reported as mean \pm SD units (for JM) and milligrams per deciliter (for BC). STB concentrations are reported as mean \pm SD milligrams per deciliter. The bilirubin concentration conversion factor from milligrams per deciliter to micromoles per liter $(\mu \mathrm{M})$ is 17.1 .

TcB values were correlated with STB concentrations and analyzed by linear regression using the method of least squares. $95 \%$ confidence intervals (CI) were calculated for slopes and intercepts. Correlations were considered significant when $p<0.05$.

Mean bias \pm imprecision of measurements by each instrument was calculated by the method of Bland and Altman (26). The JM readings were converted to milligrams per deciliter using the linear regression equations generated from each site.

\section{RESULTS}

Figure $3 A$ shows the time course of bilirubin accumulation as measured with the JM at the back of the head, upper back, and lower back of homozygous GR pups and the back of the head of normal, wild-type (control) Wistar pups up to the first 


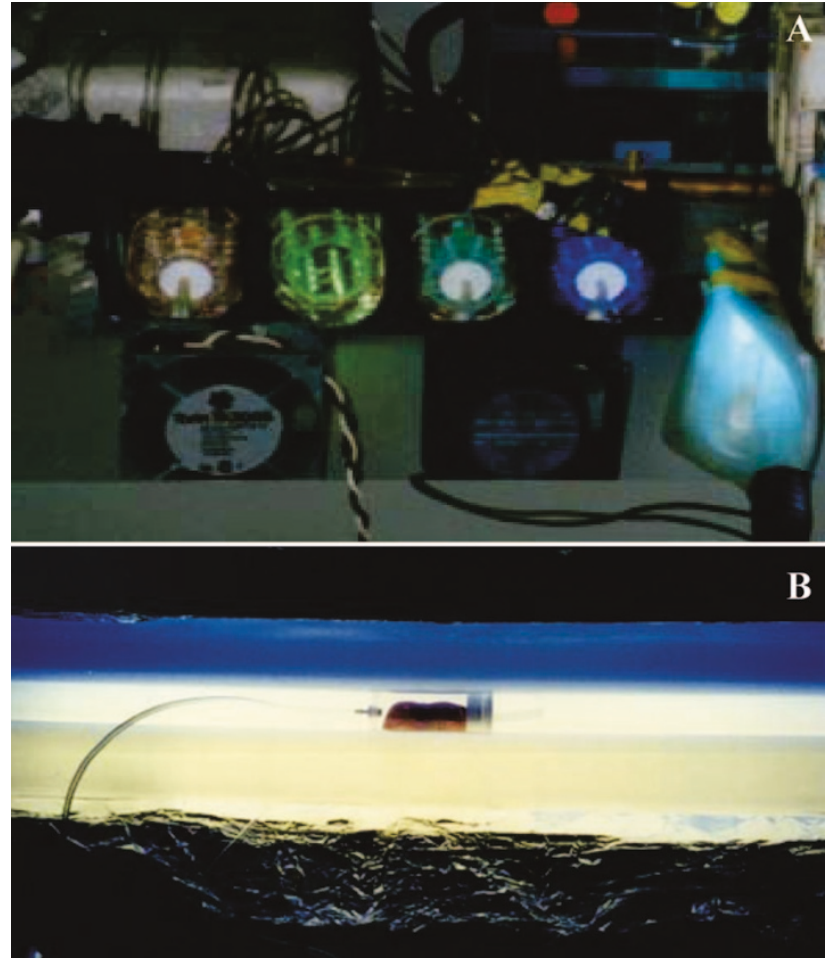

Figure 2. Devices used for the delivery of light to rat pups. (A) LED devices delivering $360^{\circ}$ of white, green, blue green, and blue light, and the Ohmeda BiliBlanket. (B) Fluorescent tubes delivering $360^{\circ} \mathrm{BB}$ and $\mathrm{CW}$ light to a pup in the chamber, which received a flow of $60 \mathrm{~mL}$ air/min. The set-ups were air cooled with room air to $23 \pm 1^{\circ} \mathrm{C}$.

$96 \mathrm{~h}$ of life. Maximum values were reached approximately $30-40 \mathrm{~h}$ after birth. Readings taken at the head of the GR pups showed a clear trend of rapidly increasing values in the first $24 \mathrm{~h}$, then a slow rise during the next $6 \mathrm{~h}$, reaching a maximum level of 12 by $30 \mathrm{~h}$ followed by a slight decline after $48 \mathrm{~h}$. The upper and lower back measurements showed a similar trend, but with lower plateau levels (approximately 8 units), greater standard
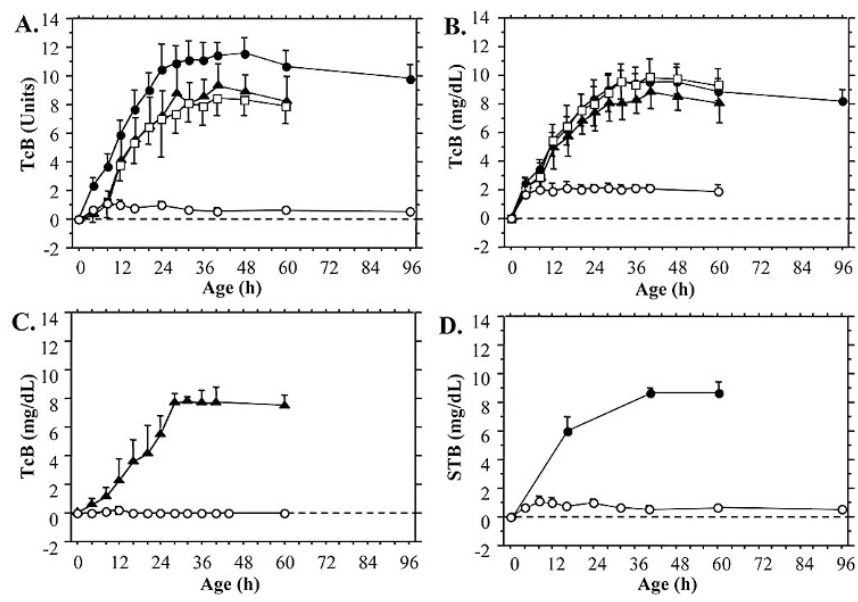

Figure 3. Time course of bilirubin accumulation in newborn GR and control Wistar pups as a measured with: (A) JM at the head, lower back, and upper back; $(B) \mathrm{JM}$ readings converted to $\mathrm{mg} / \mathrm{dL} ;(C) \mathrm{BC}$ at the lower back. Measurements made on Wistar pups (control) using both devices are shown only for the head. $(D)$ Time course of STB levels as measured by the UB Analyzer in the GR and wild-type Wistar pups (controls). Each time point represents $\mathrm{TcB}$ measurements on a minimum of 10 pups. deviations, and an initial lag phase from 0 to $8 \mathrm{~h}$. The TcB readings on the head of the control animals leveled at 1.8 units.

Figure $3 C$ shows the time course of bilirubin accumulation as measured with the $\mathrm{BC}$ at the lower back of homozygous GR and normal Wistar pups over the first $60 \mathrm{~h}$ of life. A similar $\mathrm{TcB}$ profile to that observed with the JM at the same site (Fig. 3A) was found with a maximum reading of approximately $7.8 \mathrm{mg} / \mathrm{dL}$. Plateau values were reached within $28 \mathrm{~h}$ after birth. It is noteworthy that, in contrast to JM measurements on the head, measurements taken at the back with the $\mathrm{BC}$ also showed an 8-h lag phase. The measurements on the lower back of the control Wistar pups remained stable $(0.0$ $\mathrm{mg} / \mathrm{dL}$ ) after an initial, slow rise.

The time courses for STB accumulation in separate sets of $n \geq 3$ newborn GR and Wistar pups are shown in Figure 3D. The profile of the STB concentration was similar in this group to the TcB groups in that they reached a plateau of approximately $8 \mathrm{mg} / \mathrm{dL}$ at $40 \mathrm{~h}$ of age.

Figure 4, $A-C$ displays the correlation between STB and $\mathrm{TcB}$ measurements made with the JM at the head, upper back, and lower back of homozygous GR pups, respectively. TcB measurements with the $\mathrm{BC}$ taken at the lower back of homozygous pups are shown in Figure $4 D$. The regression equations from Figures $4, A-C$, and $6, A-C$ (see Table 3 ), were used to convert the arbitrary JM readings to milligrams per deciliter for the Bland-Altman analyses shown in Figures 5, $A-C$, and $7, A-C$, respectively.

Figure 5 shows the mean bias \pm imprecision of $\mathrm{TcB}$ measurements taken with the JM at the head, lower back, and upper back, and with the BC.

The age and weight of rat pups at the time of light exposure was $40.5 \pm 12.6 \mathrm{~h}$ and $6.5 \pm 0.6 \mathrm{~g}$, respectively. Figure 6 illustrates the effect of $4 \mathrm{~h}$ of exposure to six different light sources, each with its own blue light irradiance, on the correlation between $\mathrm{TcB}$ measurements at various sites with the $\mathrm{JM}$ and BC and STB determinations.

Figure 7 shows the effect of $4 \mathrm{~h}$ of light exposure with various light sources on the mean bias \pm imprecision of $\mathrm{TcB}$ measurements taken with the JM at the head, lower back, and upper back, and with the $\mathrm{BC}$.
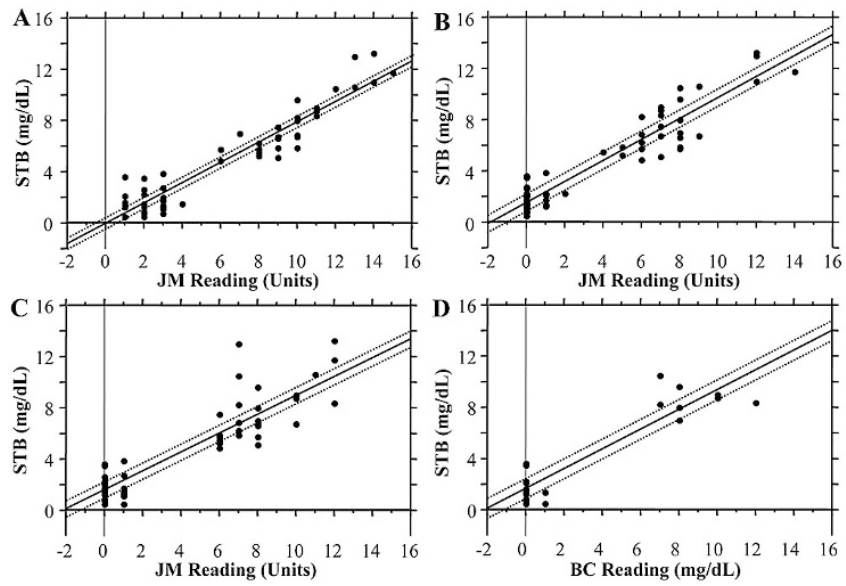

Figure 4. Correlations between STB concentrations $(\mathrm{mg} / \mathrm{dL})$ and $\mathrm{TcB}$ measurements with the JM taken at the $(A)$ head, $(B)$ upper back, $(C)$ lower back, and $(D)$ with the $\mathrm{BC}$ measured at the lower back (see Table 3 ). 

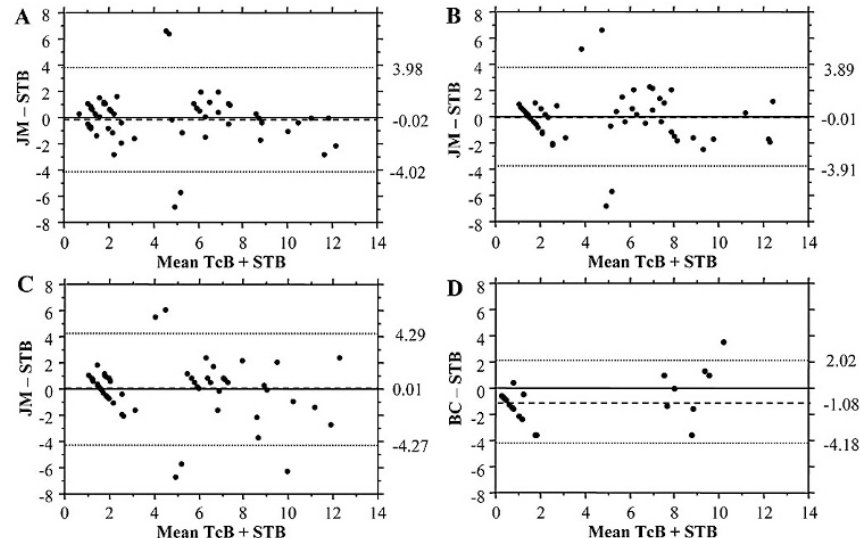

Figure 5. Mean bias \pm imprecision of TcB measurements of the JM (converted to $\mathrm{mg} / \mathrm{dL}$ ) at the $(A)$ head, $n=60,(B)$ upper back, $n=60$, and (C) lower back, $n=60$, and $(D)$ with the BC at the lower back $n=29$.
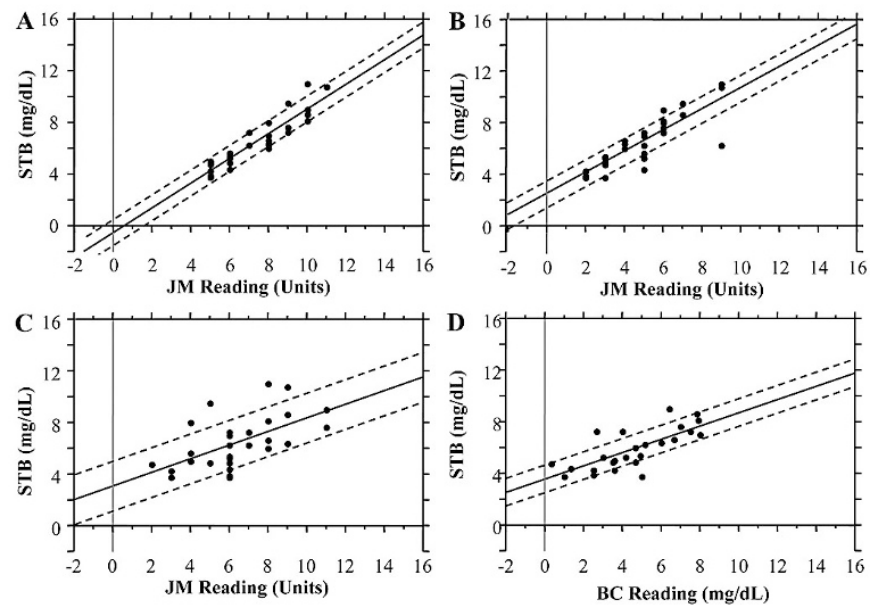

Figure 6. Effect of light exposure on correlations between STB concentrations $(\mathrm{mg} / \mathrm{dL})$ and $\mathrm{JM} \mathrm{TcB}$ measurements taken at the $(A)$ head, $(B)$ upper back, and $(C)$ lower back. The $\mathrm{BC}$ measured at the $(D)$ lower back after exposure to six different light sources (see Table 3).
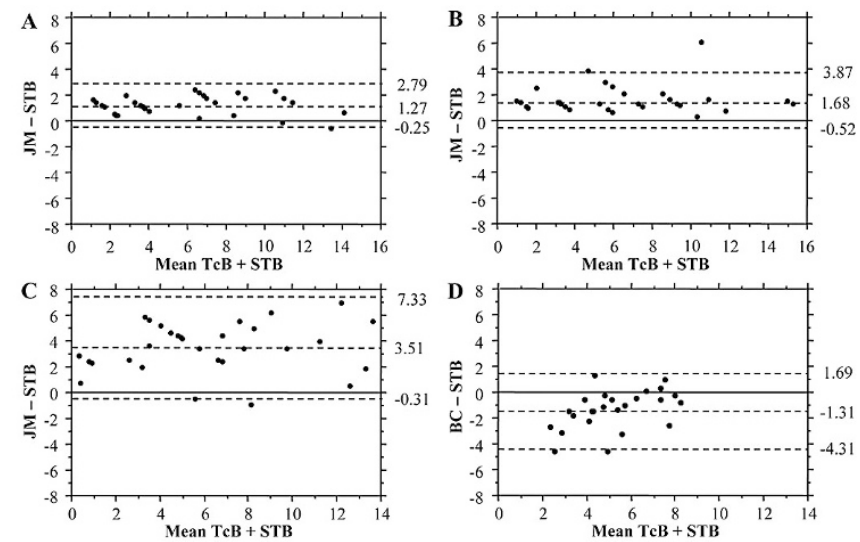

Figure 7. Mean bias \pm imprecision of TcB measurements and STB concentrations $(\mathrm{mg} / \mathrm{dL})$ with the JM at the $(A)$ head, $n=31,(B)$ upper back, $n=31$, and $(C)$ lower back, $n=31$, and $(D)$ with the BC at the lower back, $n=25$ after exposure to six different light sources.

Table 1 summarizes the mean bias \pm imprecision of $\mathrm{TcB}$ measurements taken by both instruments before and after light exposure.
Table 2 shows the effect of $4 \mathrm{~h}$ of light exposure by different light sources on the mean $\pm \mathrm{SD}$ of TcB measurements and STB concentrations $(\mathrm{mg} / \mathrm{dL})$ with the JM at the head and with the $\mathrm{BC}$ at the lower back. The efficacy of a given light source to decrease STB levels during the $4 \mathrm{~h}$ exposure time is expressed as \%STB left and summarized in Figures 5 and 7 and Table 2.

\section{DISCUSSION}

This is the first report on performing $\mathrm{TcB}$ measurements on small $(<10 \mathrm{~g})$ animals. The objective of this study was to determine whether $\mathrm{TcB}$ measurements can be used as an index of STB levels in the neonatal GR and determine the effect of light exposure on these measurements $(19,20)$. With the present study, we demonstrate (Fig. 3) for the first time that $\mathrm{TcB}$ measurements can be successfully made on newborn rat pups weighing as little as $7 \mathrm{~g}$ and that measurements made at the appropriate site closely parallel STB concentrations during the first few weeks of life, and most likely thereafter, if the animal's fur is removed. TcB measurements with two commercially available devices show that the jaundice levels increase rapidly during the first $24 \mathrm{~h}$, plateau or slightly decrease from 30 to $96 \mathrm{~h}$ of life as elegantly described by Johnson et al. (12) using STB measurements. The plateau is likely to be due to the equilibration between bilirubin production, catabolism, derivitization by mixed function mono-oxygenase (27), and biliary excretion of unconjugated bilirubin into the intestine $(14,15)$ and, possibly, the enterohepatic circulation.

It is interesting to note that, in contrast to the STB, the TcB time course measurements with both devices display an initial (8-h) lag phase for the back sites only, most likely representing the loading of subdermal tissues with bilirubin as is demonstrated on a total body scale by the cephalocaudal progression of jaundice seen in human neonates (28).

The regression analysis results displayed in Figure 4 (see Table 3) show that $\mathrm{TcB}$ measurements with both devices and all sites correlated strongly with STB measurements in homozygous GR during the first week of life $(r=0.91-0.95, p$ $\leq 0.0001$ ). Also, the slopes fell within a fairly narrow range of $0.74-0.82$, with fairly narrow CI. Only the intercept for the head measurements intersected the origin, whereas the other sites measurements were similar and ranged from 1.51 to 1.65 . On the basis of these results with untreated (no light exposure) animals, neither of the devices showed clear superiority over the other. However, we tend to favor the JM device, because it allows measurements of TcB at three sites, its head measurements intersected the origin and yielded the closest correlation with STB, and finally was more economical since it does not require costly disposables.

The Bland-Altman analyses in Figure 5 and Table 1 demonstrated that the bias of the JM device was similar between measurements taken at the lower back $(+0.01)$, upper back $(-0.01)$, and head $(-0.02)$. Mean bias of the BC device was -1.08 . Imprecision of both devices increased at STB $>8 \mathrm{mg} / \mathrm{dL}$. In addition, bias and imprecision slightly increased for readings taken after light exposure by both instruments. 
Table 1. Mean bias \pm imprecision (2 SD) of TcB measurements with JM at the head, upper back, and lower back, and with the BC pre- and post-light exposure compared with STB measurements

\begin{tabular}{|c|c|c|c|c|}
\hline Condition & \multicolumn{3}{|c|}{$\mathrm{JM}$ (mg/dL) } & $\begin{array}{l}\text { BC (mg/dL) } \\
\text { Lower Back }\end{array}$ \\
\hline Pre-exposure & $-0.02 \pm 3.99(n=60)$ & $-0.01 \pm 3.90(n=60)$ & $0.01 \pm 4.28(n=60)$ & $-1.08 \pm 3.10(n=29)$ \\
\hline
\end{tabular}

Table 2. Pre- and post-4 h of exposure in the dark and with different light sources (blue, white, blue green (BG), and green LED; BiliBlanket; and fluorescent special blue $(B B)$ and cool white $(C W)$ tubes) on TcB measurements with JM at the head, and with the BC at the lower back of homozygous GR pups

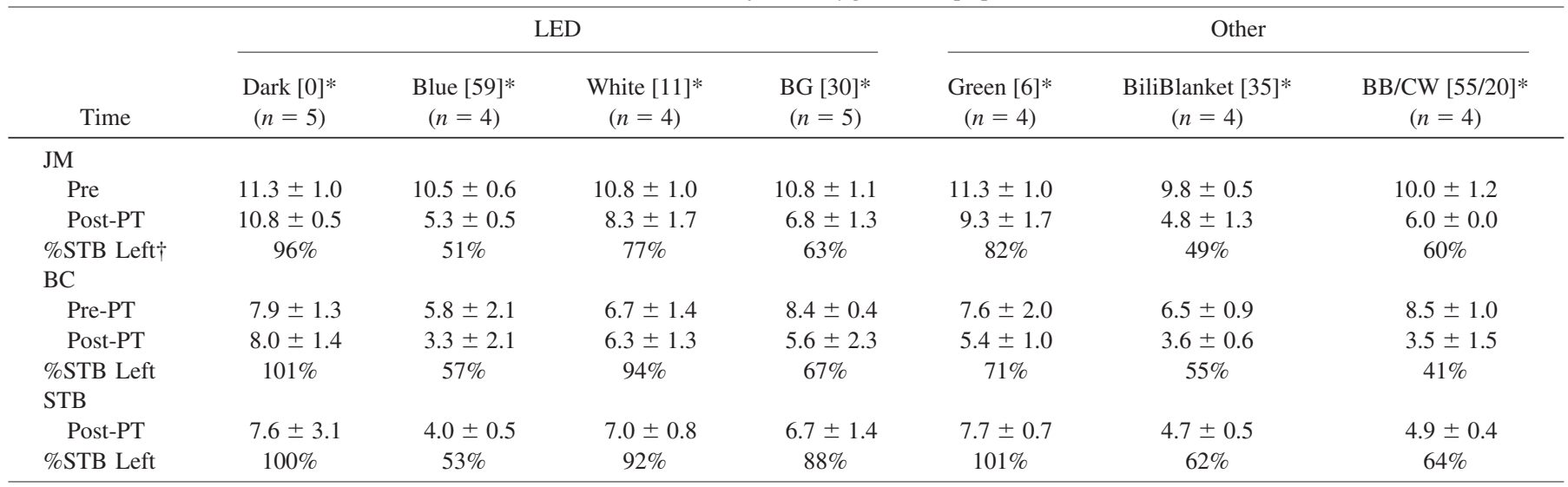

STB concentrations $(\mathrm{mg} / \mathrm{dL})$ were measured only after $4 \mathrm{~h}$ of light exposure.

* Light intensity $\left[\mu \mathrm{W} / \mathrm{cm}^{2} / \mathrm{nm}\right]$ was measured using the BiliBlanket Meter I (Ohmeda Medical, Inc).

$\dagger$ STB calculated from the regression equation from Figure $4 A$ (head).

It has been suggested that $\mathrm{TcB}$ measurements in human subjects receiving phototherapy are less than useful. However, Myara et al. (20) exposed jaundiced neonates to $3 \mathrm{~h}$ of intensive phototherapy and found that the TcB decreased from 20 to $9 \mathrm{mg} / \mathrm{dL}$ and slowly rebounded up to $9 \mathrm{~h}$ thereafter. Hegyi et al. (19) also used TcB measurements to demonstrate the response of cutaneous bilirubin to different colors of light during phototherapy. Thus, it is not surprising that TcB measurements in this small animal model can also serve as a reliable index for STB concentrations. The small size of the animal, with the consequent smaller distance between circulation and the skin, may contribute to better correlation between TcB and STB. TcB measurements could be very useful during and after phototherapy to study noninvasively the dynamics of this process in the skin for the purpose of optimizing the various aspects of phototherapy regimens, such as light quality, intensity, duration of exposure, efficacy of intermittent exposures, and varying the duty cycle of the light.

Therefore, in preliminary experiments with light sources emitting different qualities and intensities of effective bilirubin altering light, we also made measurements of TcB and STB in light-exposed GR to evaluate the applicability of TcB measurements after light treatment of limited 4-h duration. The results should only be judged qualitatively, as any change in these parameters, including animal size is likely to yield different quantitative results (29). Prelight exposure STB measurements in the same animals as the postlight animals was not possible, because this measurement involves the death of the animal, which is the reason why fairly accurate, reproducible, and noninvasive $\mathrm{TcB}$ are so valuable for research activ- ities with newborn rats. However, using the mean \pm SD of $7.6 \pm 3.1 \mathrm{mg} / \mathrm{dL}$ for the age-matched animals protected from light allowed us to calculate the STB left after light exposure. The results in Table 2 demonstrate that $4 \mathrm{~h}$ of light exposure effectively decreased the TcB as well as the STB values in all groups, albeit to a variable extent, presumably based on the quality and intensity of the light. In general, we can conclude that JM and $\mathrm{BC}$ measurements agreed fairly closely and that both approximated the STB measurements with regard to the $\%$ STB left for most light sources, except for blue green and green LEDs. Our results are valid for up to $4 \mathrm{~h}$ of light exposure and different results may be found with longer light exposure times. This topic clearly needs further detailed examination.

It is important to recognize that $\mathrm{TcB}$ devices have been designed to estimate STB by measuring the presence of bilirubin in the skin, both capillary and extravascular, relative to that found in the central circulation. The devices do not offer a true or absolute concentration of bilirubin in the skin itself nor are they intended to. Previous work by others $(13,16)$ has shown that rat skin bilirubin concentrations are 10-20\% lower than those found in the plasma, which in turn have been shown to reflect STB. Bessard et al. (16) have found bilirubin concentrations in human newborn skin to be 4-5-fold higher than those in GR skin.

It is interesting to note that, in contrast to measurements in human subjects, the magnitude of the arbitrary values reported by the JM closely parallel the STB concentrations. In other words, the regression lines in Table 3 all nearly intercept the origin. In contrast, the y-intercept found with human new- 
borns ranges from 8 to 13 (30). This observation could indicate that the human skin contains nonbilirubin yellow pigments that absorb light in the absence of significant concentrations of bilirubin skin and/or the underlying blood vessels. Alternatively, the small size and the degree of transparency of the pup could have affected, to a fortuitous extent, the proportion of the incident light reflected back into the device.

When the regression equations in Figure 6 are compared with those in Figure 4 (Table 3), it appears that JM measurements at the head are the most similar, whereas, for all other areas, the correlations between TcB and STB measurements were lower. Other body sites were also considered but were not rugged or large enough for accommodating the surface area and pressure of the meters. The chest area was deemed too fragile to withstand the pressure even with the present protective jig. Older animals with a greater choice of sites after the fur is removed, and the ear may also become an appropriate site.

Until the present efforts, $\mathrm{TcB}$ measurements have not been translated for use in small animals, where they can offer significant experimental advantages over invasive STB measurements. TcB measurements can circumvent the technical difficulties and consequential physiologic effects involved in the invasive drawing of serial blood samples of sufficient volume for STB measurements (12). Furthermore, the withdrawal of blood affects the very system that is being studied because it decreases the already small blood volume, which is the primary source of STB. In addition, blood sampling could artificially elevate STB levels by producing extravascular hematomas. TcB measurements could also be useful identifying, early after birth, which animals are homozygous $(\mathrm{j} / \mathrm{j}) \mathrm{GR}$ and which are most likely to develop kernicterus, without having to resort to invasive blood drawing (12). Furthermore, the technique is expected to be a valuable tool for experimental GR models of kernicterus, especially newborns, after administration of displacing sulfonamides and during sepsis, conditions that have been shown to decrease STB and are expected to increase $\mathrm{TcB}$ values $(12,31)$.

Because this is the first study reporting $\mathrm{TcB}$ values in newborn rats and no studies with other animal species of similar small size $(<10 \mathrm{~g})$ have been reported, we cannot compare our results to those of others.

Table 3. Comparison of linear regression analysis of $T c B$ and STB concentrations $(\mathrm{mg} / \mathrm{dL}$ ) measurements with JM (arbitrary units) at the head, upper back, and lower back and with the $B C(\mathrm{mg} / \mathrm{dL})$ at the lower back of homozygous GR pups pre-and post 4 h of exposure with different light sources

\begin{tabular}{ccc}
\hline \multicolumn{1}{c}{ TcB device } & Exposure & \multicolumn{1}{c}{ Regression equation $(r, n)$} \\
\hline JM (head) & Pre- & STB $=0.79(\mathrm{JM})-0.01(r=0.95, n=60)$ \\
& Post- & STB $=0.96(\mathrm{JM})-0.57(r=0.93, n=31)$ \\
JM (upper back) & Pre- & STB $=0.82(\mathrm{JM})+1.51(r=0.95, n=60)$ \\
& Post- & $\mathrm{STB}=0.82(\mathrm{JM})+2.47(r=0.85, n=31)$ \\
$\mathrm{JM}$ (lower back) & Pre- & $\mathrm{STB}=0.74(\mathrm{JM})+1.60(r=0.91, n=60)$ \\
& Post- & $\mathrm{STB}=0.53(\mathrm{JM})+3.07(r=0.60, n=31)$ \\
$\mathrm{BC}$ (lower back) & Pre- & $\mathrm{STB}=0.77(\mathrm{BC})+1.65(r=0.93, n=29)$ \\
& Post- & $\mathrm{STB}=0.52(\mathrm{BC})+3.51(r=0.74, n=25)$ \\
\hline
\end{tabular}

On the basis of the present studies, we conclude that TcB measurements can be easily made on animals weighing as little as $7 \mathrm{~g}$. Furthermore, the results with both devices strongly correlate with STB measurements, which means that serial noninvasive $\mathrm{TcB}$ measurements can be used as fairly accurate, precise, and reliable substitutes for the invasive STB measurements.

Acknowledgments. The authors thank SpectRx Inc. for the use of the BiliCheck System with a generous supply of BiliCal calibration tips.

\section{REFERENCES}

1. Dennery PA, Seidman DS, Stevenson DK 2001 Neonatal hyperbilirubinemia. N Engl J Med. 344:581-590

2. Crigler Jr, JF Najjar VA 1952 Congenital familial nonhemolytic jaundice with kernicterus. Pediatrics 10:169-180

3. Dennery PA, McDonagh AF, Spitz DR, Rodgers PA, Stevenson DK 1995 Hyperbilirubinemia results in reduced oxidative injury in neonatal Gunn rats exposed to hyperoxia. Free Radic Biol Med 19:395-404

4. Stevenson DK, Bartoletti AL, Ostrander CR, Johnson JD 1979 Pulmonary excretion of carbon monoxide in the human newborn infant as an index of bilirubin production: III. Measurement of pulmonary excretion of carbon monoxide after the first postnatal week in premature infants. Pediatrics 64:598-600

5. Stevenson DK, Bartoletti AL, Ostrander CR, Johnson JD 1979 Pulmonary excretion of carbon monoxide in the human infant as an index of bilirubin production. II. Infants of diabetic mothers. J Pediatr 94:956-958

6. Slusher TM, Angyo IA, Bode-Thomas F, Akor F, Pam SD, Adetunji AA, McLaren DW, Wong RJ, Vreman HJ, Stevenson DK 2004 Transcutaneous bilirubin measurements and serum total bilirubin levels in indigenous African infants. Pediatrics 113:1636-1641

7. Bhutani VK, Gourley GR, Adler S, Kreamer B, Dalin C, Johnson LH 2000 Noninvasive measurement of total serum bilirubin in a multiracial predischarge newborn population to assess the risk of severe hyperbilirubinemia. Pediatrics 106:E17

8. Dai J, Parry DM, Krahn J 1997 Transcutaneous bilirubinometry: its role in the assessment of neonatal jaundice. Clin Biochem 30:1-9

9. Maisels MJ, Kring E 1998 Length of stay, jaundice, and hospital readmission. Pediatrics 101:995-998

10. Kass PH, Lopezflores A, Farver TB 1998 Use of transcutaneous bilirubinometry as a screening and monitoring instrument for hyperbilirubinemia in cats and dogs. Canine Pract 23:12-15

11. Gunn CH 1938 Hereditary acholuric jaundice in new mutant strain of rats. J Hered 29:137-139

12. Johnson L, Sarmiento F, Blanc WA, Day R 1959 Kernicterus in rats with an inherited deficiency of glucuronyl transferase. Am J Dis Child 97:591-608

13. Schmid R, Axelrod J, Hammaker L, Swarm RL 1958 Congenital jaundice in rats, due to a defect in glucuronide formation. J Clin Invest 37:1123-1130

14. Swarm RL 1971 Congenital Hyperbilirubinemia in the Rat: An Animal Model for the Study of Hyperbilirubinemia. National Academy of Science, Washington, DC, pp $149-160$

15. Kotal P, Van der Veere CN, Sinaasappel M, Elferink RO, Vítek L, Brodanová M, Jansen PL, Fevery J 1997 Intestinal excretion of unconjugated bilirubin in man and rats with inherited unconjugated hyperbilirubinemia. Pediatr Res 42:195-200

16. Bessard G, Chouraqui JP, Rambaud P 1982 A spectrophotometric assay of skin bilirubin in adult Gunn rats. Biol Neonate 42:59-65

17. Dai J, Krahn J, Parry DM 1996 Clinical impact of transcutaneous bilirubinometry as an adjunctive screen for hyperbilirubinemia. Clin Biochem 29:581-586

18. Stevenson DK, Rodgers PA, Vreman HJ 1989 The use of metalloporphyrins for the chemoprevention of neonatal jaundice. Am J Dis Child 143:353-356

19. Hegyi T, Graff M, Zapanta V, Hiatt IM, Sisson TR 1986 Transcutaneous bilirubinometry. III. Dermal bilirubin kinetics under green and blue light phototherapy. Am J Dis Child 140:994-997

20. Myara A, Sender A, Valette V, Rostoker C, Paumier D, Capoulade C, Loridon F, Bouillie J, Milliez J, Brossard Y, Trivin F 1997 Early changes in cutaneous bilirubin and serum bilirubin isomers during intensive phototherapy of jaundiced neonates with blue and green light. Biol Neonate 71:75-82

21. Yamanouchi I, Yamauchi Y, Igarashi I 1980 Transcutaneous bilirubinometry: preliminary studies of noninvasive transcutaneous bilirubin meter in the Okayama National Hospital. Pediatrics 65:195-202

22. Yamauchi Y, Yamanouchi I 1988 Transcutaneous bilirubinometry. Evaluation of accuracy and reliability in a large population. Acta Paediatr Scand 77:791-795

23. Rubaltelli FF, Gourley GR, Loskamp N, Modi N, Roth-Kleiner M, Sender A, Vert P 2001 Transcutaneous bilirubin measurement: A multicenter evaluation of a new device. Pediatrics 107:1264-1271

24. Nakamura H, Yonetani M, Uetani Y, Funato M, Lee Y 1992 Determination of serum unbound bilirubin for prediction of kernicterus in low birthweight infants. Acta Paediatr Jpn 34:642-647

25. Vreman HJ, Wong RJ, Stevenson DK, Route RK, Reader SD, Fejer MM, Gale R, Seidman DS 1998 Light-emitting diodes: a novel light source for phototherapy. Pediatr Res 804-809 
26. Bland JM, Altman DG 1986 Statistical methods for assessing agreement between two methods of clinical measurement. Lancet 1:307-310

27. Cohen AN, Kapitulnik J, Ostrow JD, Zenone EA, Cochrane C, Celic L, Cheney H 1985 Effects of phenobarbital on bilirubin metabolism and its response to phototherapy in the jaundiced Gunn rat. Hepatology 5:310-316

28. Hegyi T, Hiatt IM, Gertner I, Indyk L 1981 Transcutaneous bilirubinometry. The cephalocaudal progression of dermal icterus. Am J Dis Child 135:547-549
29. Vreman HJ, Wong RJ, Stevenson DK 2004 Phototherapy: current methods and future directions. Semin Perinatol 28:326-333

30. Hannemann RE, Schreiner RL, DeWitt DP, Norris SA, Glick MR 1982 Evaluation of the Minolta bilirubin meter as a screening device in white and black infants. Pediatrics 69:107-109

31. Schutta HS, Johnson L 1969 Clinical signs and morphologic abnormalities in Gunn rats treated with sulfadimethoxine. J Pediatr 75:1070-1079 